\section{REDER, UNA REVISTA PARA AVANZAR EN LA REDUCCIÓN DEL RIESGO DE DESASTRES Y EL MEJORAMIENTO DEL BIENESTAR HUMANO}

Carmen Paz Castro Correa ${ }^{1,2 *}$

\section{RESUMEN}

Para reducir el riesgo de desastres y abordar sus causas, se debe contar con estrategias de desarrollo basadas en procesos de transformación que disminuyan la vulnerabilidad social y aumenten las capacidades de gestión. En Latinoamérica así como en el resto del mundo, actualmente enfrentamos una creciente complejidad en los patrones socio-espaciales del riesgo así como en sus factores explicativos, circunstancias que demandan estudios más profundos y a la vez comprehensivos. En este sentido, el siguente prólogo introduce el surgimiento de la Revista de Estudios Latinoamericanos sobre Reducción del Riesgo de Desastres (REDER) y busca posicionar su aporte a la región desde una perspectiva multidimensional e interdisciplinaria.

\section{PALABRAS CLAVE}

Reducción del riesgo de desastres, Gestión del riesgo, Vulnerabilidad, Resiliencia

\section{ABSTRACT}

To reduce disaster risks and address their causes, development strategies based on transformation processes that reduce social vulnerability and increase management capacities should be developed. As in Latin America and in the rest of the world, we are currently facing a growing complexity in the socio-spatial patterns of risk as well as in their explanatory factors, circumstances that require deeper and at the same time, comprehensive studies. In this sense, the following prologue introduces the emergence of the Journal of Latin American Studies on Disaster Risk Reduction (Revista de Estudios Latinoamericanos sobre Reducción del Riesgo de Desastres - REDER) and seeks to position its contribution to the region from a multidimensional and interdisciplinary perspective.

\section{KEYWORDS}

Disaster risk reduction, risk management, vulnerability, resilience, holistic perspective

\author{
1. Departamento de \\ Geografía, CITRID, \\ Universidad de Chile, \\ Santiago, Chile.
2. Directora, Revista de Estudios Latinoamericanos sobre Reducción del Riesgo de Desastres (REDER), Santiago, Chile.

*Autor de correspondencia: cpcastro@uchilefau.cl

\section{Formato cita}

Recomendada (APA): Castro Correa, C.P. (2017). REDER, una revista para avanzar en la reducción del riesgo de desastres y el mejoramiento del bienestar humano. REDER, 1(1), pp.1-5

\section{$(\Theta)(\mathbb{D} \otimes$}

Todos los artículos publicados en REDER siguen una política de Acceso Abierto y se respaldan en una Licencia CreativeCommons Atribución-NoComercial 4.0 Internacional.

Revista de Estudios Latinoamericanos sobre Reducción del Riesgo de Desastres (REDER)

Diseño: Lupe Bezzina Tipografías: Hospital 


\section{PRÓLOGO}

El riesgo de desastres se está estableciendo cada vez con mayor fuerza como objeto de estudio en los intereses de investigación, así como en las agendas de gobiernos y organismos internacionales alrededor del mundo. La ocurrencia de eventos adversos ha causado daños humanos, económicos, sociales y ambientales lamentables, generando la necesidad de mejorar los sistemas de gestión y reducción de riesgos y planteando retos importantes a la comunidad académica y a las instituciones nacionales e internacionales que han tenido que adaptarse y transformarse en los últimos años para mejorar la gestión y reducción de los riesgos. A pesar de los esfuerzos continuos para gestionar los riesgos, a través de un trabajo interinstitucional coordinado y un abordaje multidimensional, persiste un proceso acelerado de construcción del riesgo.

Este proceso se asocia principalmente a los estilos de crecimiento económico que no consideran el resguardo de la sostenibilidad ambiental y que han traído consigo intensas y aceleradas modificaciones en los patrones de urbanización e incremento en los niveles de exposición de la población y sus bienes a amenazas de diferentes orígenes, así como la mantención y en muchos casos el aumento de la vulnerabilidad de la población. En este contexto, los procesos urbanos que en general manifiestan una ocupación del territorio de forma desregulada y la presión por realizar proyectos de inversión que entreguen ganancias a corto plazo, entre otros factores, contribuyen a la agudización de los contextos de fragilidad social, territorial y ambiental, incubando las condiciones necesarias para la concreción de futuros desastres. Es necesario considerar aquellos patrones cambiantes de la percepción social del riesgo, que tienen el potencial de frenar o incluso contrarrestar, los esfuerzos hechos para mejorar la gestión del riesgo, fruto de la experiencia histórica.

Estas condiciones deben ser identificadas, evaluadas y reconocidas como causas subyacentes del riesgo, y así proceder a tomar acciones de prevención y adaptación que trasciendan las medidas estructurales enfocadas solamente a mitigar la amenaza. Entre estas acciones se hace necesario abordar aquellos aspectos relacionados a la disminución de la vulnerabilidad y de la exposición de la población y sus bienes, así como a mejorar la preparación de la comunidad y su involucramiento en la toma de decisiones de la gestión del riesgo. La comunidad científica internacional ha mostrado un amplio consenso en cuanto a que la probabilidad de una sociedad a sufrir daños debido al impacto de un fenómeno externo radica mayormente en el conjunto de condiciones económicas, sociales y ambientales existentes en el momento en que se produce un evento adverso, y no exclusivamente en la magnitud del evento físico-natural o antrópico que gatilla el desastre.

Es evidente entonces que para reducir el riesgo de desastres y abordar sus causas, se debe contar con estrategias de desarrollo basadas en procesos de transformación que disminuyan la vulnerabilidad social y aumenten las capacidades de gestión y adaptación. En este sentido, el concepto de resiliencia cobra cada día más fuerza y ha sido objeto de múltiples abordajes que han generado recomendaciones a los Estados sobre la necesidad de focalizar recursos en mejora de las capacidades de la población y de los territorios para enfrentar y reponerse del impacto de eventos adversos, así como para adaptarse a las nuevas condiciones. Un sistema social con alta resiliencia podría ser capaz de absorber la crisis, mantener la funcionalidad y transformarse para que la comunidad pueda fortalecerse y sea capaz de enfrentar eficiente y efectivamente estos fenómenos a futuro.

En las últimas décadas los acuerdos internacionales han contribuido a fortalecer la agenda, promover reflexiones e intercambios de conocimiento, y a coordinar esfuerzos. El Marco de Acción de Hyogo - MAH (2005 - 2015) constituyó un hito en cuanto a los avances en reducción del riesgo de desastres (RRD) a nivel internacional, procurando el mejoramiento de la resiliencia de las naciones y las comunidades ante los desastres. Sin embargo, las evaluaciones del proceso de implementación del MAH no fueron muy alentadoras, revelando un importante incremento en el número e intensidad de los desastres asociados a fenómenos de origen natural. Frente a esto, se ha argumentado que una de las debilidades del modelo de acción recae en que mantuvo su énfasis en la preparación para la respuesta a la emergencia y el manejo del riesgo, y no en la reducción de estos o en abordar sus causas subyacentes, así como en la gestión institucional del riesgo más que en la incorporación de la comunidad en la toma de decisiones.

No obstante, la experiencia obtenida a través de los esfuerzos de implementación del MAH ha demostrado que la RRD es a largo plazo una inversión en prevención que evita o reduce pérdidas futuras. Asimismo, esa experiencia y el aprendizaje logrado constituyeron la base del nuevo Marco de Sendai para la Reducción del Riesgo de Desastre 2015 - 2030. Éste hace énfasis en la necesidad de avanzar en la prevención y reducción del riesgo y reforzar la resiliencia de las comunidades y medios 
de vida; la importancia de fomentar una implementación de carácter multisectorial y multiescalar, fortaleciendo la gobernanza del riesgo con un enfoque preventivo y centrado en las personas.

De esta forma, el Marco de Sendai (2015 - 2030) busca garantizar la continuidad del trabajo realizado a través de $\mathrm{MAH}$, pero al mismo tiempo incorporar un nuevo enfoque y objetivos para RRD a nivel internacional y local. Entre las principales modificaciones, se destaca la importancia asignada a los esfuerzos en prevención y reducción del riesgo de desastres por sobre la atención y respuesta a emergencias, reduciendo el riesgo existente y previniendo la aparición de nuevas condiciones que lo generen. Al mismo tiempo, la necesidad de trabajar a escala local, incorporando a las comunidades y a la sociedad civil de una forma continua y sistemática considerando sus saberes y prácticas, así como influir en la creación de territorios seguros a largo plazo.

\section{ENFOQUE HOLÍSTICO DEL RIESGO DE DESASTRES}

El conocimiento del riesgo de desastres ha registrado diversos cambios de paradigmas a través del tiempo, derivados de los avances en las diferentes disciplinas involucradas así como de la experiencia empírica, que han ido evolucionando hacia enfoques más holísticos y apropiados para la consideración de las diversas variables identificadas en la problemática. Estos avances se han visto reflejados en un foco de análisis más balanceado entre los fenómenos naturales y el conocimiento de las condiciones sociales y de la intervención poco sustentable del territorio, que llevan a considerar al ser humano como parte responsable del riesgo generado.

Dentro de los cambios de enfoque se aprecia también una tendencia a dejar atrás la visión fatalista de los desastres como eventos inevitables, avanzando hacia una visión más positiva, que plantea que es posible prevenir y reducir los impactos de los eventos extremos. Esto lleva a la necesidad de entender el riesgo de desastres en forma holística, tanto para la evaluación del riesgo y las problemáticas subyacentes, como para las propuestas de intervenciones prácticas para su reducción, para lo cual es necesario avanzar hacia la transdisciplina y post-disciplina, profundizando en la teoría del riesgo.

\section{PENSANDO DESDE Y PARA AMÉRICA LATINA}

El concebir el riesgo de desastres como una construcción social, refleja al mismo tiempo que las condiciones de riesgo son productos de procesos asociados al crecimiento económico mal llamado desarrollo. La acumulación de condiciones de riesgo se debe a causas de fondo que no han sido del todo estudiadas y que generan patrones de vulnerabilidad y falta de resiliencia, productos de procesos dinámicos asociados a transformaciones del territorio sin consideraciones de sostenibilidad.

En este contexto, América Latina se caracteriza por un estilo de crecimiento con fuertes desequilibrios económicos y socio-ambientales que ha agudizado la degradación del medio ambiente y la vulnerabilidad social, sin que se vea un cambio a mediano plazo que permita revertir el aumento de la inseguridad territorial que provoca estas condiciones. Esto se condice con la pérdida de vidas y el impacto económico que provocaron en la última década los 335 desastres asociados a fenómenos climáticos, que duplicaron las pérdidas correspondientes al periodo 1895-1995, según Naciones Unidas.

Por otra parte, las presiones generadas por las condiciones de variabilidad climática agudizan las fragilidades y desequilibrios generados por los modelos económicos imperantes. De esta forma, surge en la región la necesidad de integrar las visiones y objetivos para alcanzar un desarrollo sostenible, fortaleciendo las capacidades institucionales y la gobernanza en nuestros países, así como la necesidad de incorporar un enfoque de intervención multiescalar y multidimensional en el territorio, que es el eje donde convergen la planificación del desarrollo sostenible y la gestión y reducción del riesgo de desastres.

\section{LOS RETOS DE LA GESTIÓN DEL RIESGO DE DESASTRES}

La gestión del riesgo ha mantenido en general un enfoque reactivo y correctivo, centrado en la atención de la emergencia, sin incluirse en forma efectiva y vinculante en las políticas e instrumentos de la planificación territorial y sin incorporar a la comunidad en forma efectiva en la prevención y en general, en todo el proceso de RRD.

En este contexto, la toma de decisiones en gestión del riesgo, debe dar cuenta de la necesidad de incorporar el aprendizaje social y los saberes ancestrales en la gestión local, reconociendo asimismo la percepción social del riesgo como una variable fundamental para lograr procesos efectivos de gobernanza reflexiva. 
Por otra parte, existen diversas dificultades tanto de índole teórico como metodológico para avanzar en los estudios asociados a la reducción y gestión del riesgo, que tienen en parte relación con los diferentes niveles de avance en los aportes de las distintas disciplinas involucradas, la falta de bases de datos territoriales y de confiabilidad de esta información, así como de actualización permanente, además de la problemática de tener que trabajar con una gran cantidad de variables de diversa naturaleza que implica un desafío para su evaluación.

Actualmente enfrentamos una creciente complejidad de los patrones socio-espaciales del riesgo así como de sus factores explicativos, circunstancias que demandan estudios más holísticos y transdisciplinarios. Es imperante avanzar en una investigación aplicada que permita proponer políticas eficaces y elaborar programas e instrumentos dirigidos a mejorar la seguridad de nuestros países.

\section{LA CONTRIBUCIÓN DE REDER}

La Revista de Estudios Latinoamericanos sobre Reducción del Riesgo de Desastres (REDER) busca difundir conocimientos y aplicaciones que contribuyan al avance del estado del arte en temas vinculados a la generación y gestión del riego de desastres y su traspaso a las prácticas locales, regionales y nacionales.

La revista destaca la necesidad de contar con instancias de colaboración internacional que contribuyan a la integración de los países de la región y del mundo, con el objetivo de compartir y colaborar en el desarrollo del conocimiento asociado al riesgo de desastres, así como en la generación de políticas públicas. En el ámbito aplicado, destacamos la importancia de la conformación de Plataformas Regionales para la RRD, las cuales han contribuido decididamente a coordinar y orientar los esfuerzos, integrando múltiples actores y sectores a nivel regional.

REDER propone un espacio de análisis, de estudios teóricos, prácticos e inter y transdisciplinarios en el tema del riesgo de desastres, sean estos asociados a amenazas de origen natural o antrópico, en el que se aborda el fenómeno/proceso socioambiental desde su origen y materialización en forma de riesgo y desastres hasta procesos de reconstrucción, así como todos los temas relacionados que permitan avanzar en el conocimiento y discusión científica enfocado en el mejoramiento de la seguridad y bienestar humano.

Esta revista surge a partir del interés particular de Vicente Sandoval y Claudia GonzálezMuzzio, dos investigadóres chilenos con experiencia en el extranjero, quienes apoyados por la Asociación para la Gestión y Reducción del Riesgo de Desastres en Chile (GRID-Chile), deciden emprender la tarea de organizar un Equipo Editorial y un Consejo Asesor Internacional con destacados académicos y profesionales en el área y la región, dándo forma a la revista. En este sentido, REDER se declara a sí misma una organización independiente, autónoma y sin fines de lucro.

A partir de este número inaugural, invitamos a todos los interesados e interesadas a enviar artículos a esta revista que esperamos indexar a la brevedad para que se constituya en un espacio de difusión y aporte a la comunidad científica y profesional, así como a los actores públicos y privados y al público en general, en temas de riesgo de desastres en la Región Latinoamericana y del mundo, en idioma preferentemente español.

\section{PRIMER NÚMERO}

En este número inaugural de la revista REDER tenemos el honor de contar con destacados autores que han presentado sus resultados de investigaciones recientes y reflexiones en torno a la problemática actual del riesgo de desastres, que nos invitan a conducir la investigación hacia la comprensión profunda de los factores que han incidido histórica y actualmente en la construcción del riesgo.

La profesora Adriana Allen, junto a Teresa Belkow, Carlos Escalante Estrada, Silvia de los Ríos, Marco Kamiya, Rita Lambert, Liliana Miranda, Julia Wesely y Linda Zilbert Soto, presentan los resultados preliminares del proyecto 'cLIMA sin Riesgo' realizado en Lima, Perú, y nos introducen en el análisis conceptual de las "trampas de riesgo", las cuales involucran la reproducción de riesgos cotidianos característicos del entorno urbano. El estudio se focaliza en los pequeños eventos de desastre que muchas veces son invisibilizados o bien, pasan inadvertidos, pero que afectan con gran fuerza a los sectores más desposeídos de la sociedad. Se analiza la problemática en dos barrios de la ciudad de Lima, permitiendo observar escenarios de generación de riesgo y cómo en muchos casos la inversión en reducción del riesgo no corrige el problema de fondo sino que por el contrario, refuerza la construcción y mantención de las trampas de riesgo. La Dra. Allen es profesora en la Development Planning Unit (DPU) del University College London donde lidera el programa de investigación en Justicia Ambiental, Urbanización y Resiliencia. Es co-editora del International 
Journal of Urban Sustainable Development. Su labor actual se ha centrado en evaluar la capacidad transformativa de procesos co-gestionados de gobernabilidad ambiental urbana, donde incluye la temática de la prevención y reducción del riesgo, así como la adaptación al cambio climático.

El profesor Juan Pablo Sarmiento comparte un análisis relevante sobre las agendas de desarrollo post-2015 asociadas al Marco de Sendai, a los Objetivos de Desarrollo Sostenible y al Acuerdo de París CPO 21 y los resultados que podrían esperarse de su aplicación en términos de aporte a la reducción del riesgo de desastres. Destaca la importancia de la Agenda de Desarrollo Sostenible y la necesidad de identificar las trayectorias de cambio que deben realizarse para compatibilizar las tres agendas, con el objetivo de incidir en la transformación de los factores subyacentes del riesgo de desastre. El Dr. Sarmiento es investigador y Director Asociado del Instituto de Eventos Extremos de Florida International University (FIU), así como Director del Programa de resiliencia en las Américas (USAID/OFDA). Entre otros, ha sido consultor para la Organización Panamericana de Salud (OPS), miembro del United Nations Disaster Assesment and Coordination (UNDAC) y Gerente Técnico del Programa Regional de Gestión de Riesgos, que International Resources Group (IRG) implementa en América Latina para USAID/OFDA.

La investigación del Profesor Omar Darío Cardona, ingeniero civil y académico de la Universidad Nacional de Colombia, junto a Gabriel Bernal, Daniela Zuloaga, Mario Salgado-Gálvez, Diana González, nos entrega una evaluación del riesgo sísmico en Chile y Argentina a través de un enfoque probabilista, determinando el perfil catastrófico de estos países. Este estudio permite conocer acabadamente la exposición de los activos en riesgo estableciendo valores de reposición para los principales sectores económicos de cada país, lo que constituye un aporte invaluable para la gestión preventiva del riesgo sísmico. El Dr. Cardona ha sido Director Nacional de Prevención y Atención de Desastres de Colombia, entre otros cargos profesionales, así como miembro fundador de la Red de Estudios Sociales en Prevención de Desastres de América Latina. En 2004 su trayectoria fue reconocida con el Premio Sasakawa de Naciones Unidas por su extensa contribución a la evaluación y gestión del riesgo a nivel internacional.

La integración de la problemática del patrimonio cultural al análisis del riesgo se puede analizar a través del artículo de la Profesora Bernardette Esquivel, para el caso de Centroamérica y especialmente de Costa Rica, tema escasamente tratado e incorporado en la gestión del riesgo y que se asocia a aspectos relevantes de la resiliencia de los países y las comunidades. La profesora Esquivel es arquitecta de la Universidad de Costa Rica, fue miembro de la Comisión Nacional de Patrimonio de dicho país, del Comité Científico Internacional de Arquitectura en Tierra (ISEACH) y de la Red Iberoamericana PROTERRA, así como Presidenta de la asociación de Desarrollo específica para el Patrimonio Cultural y las Artes de Santo Domingo de Heredia.

Finalmente, la contribución de los profesores Roberto Moris, Pablo Contrucci y Andrea Ortega, analiza la incorporación de la evaluación del riesgo en los instrumentos de planificación territorial, uno de los problemas focales hoy en día que ha dificultado la instalación de sistemas de gestión del riesgo prospectivos en nuestros países. Se analiza específicamente el caso de Chile en el periodo posterior al gran terremoto y tsunami de 2010. Se observó la debilidad de la planificación territorial que solo está referida a zonas urbanas y que en muchos casos está desactualizada. Se desprende del estudio que las condiciones de vulnerabilidad se han mantenido en muchas localidades pobladas de la zona afectada por el terremoto/tsunami. El profesor Moris es arquitecto de la Pontificia Universidad Católica de Chile e investigador principal del Centro de Investigación para la Gestión Integrada del Riesgo de Desastres (CIGIDEN). 